\title{
Low-field-induced spin-glass behavior and controllable anisotropy in nanoparticle assemblies at a liquid-air interface
}

\author{
Xiaoqi Liao ${ }^{1,2,3}$, Seda Ulusoy ${ }^{3}$, Rui Huang ${ }^{2}$, Erik Wetterskog ${ }^{3}$, Klas Gunnarsson ${ }^{3}$, Yu Wang ${ }^{4}$, \\ Huawei Liang ${ }^{1}$, Yu-Jia Zeng ${ }^{1 *}$, German Salazar-Alvarez ${ }^{3 *}$ and Peter Svedlindh ${ }^{3}$
}

\begin{abstract}
Stacking nanoscale-building blocks into onedimensional (1D) assemblies with collective physical properties is a frontier in designing materials. However, the formation of 1D arrays using weak magnetic fields and an in-depth understanding of their magnetic properties remain challenging. Here, low-dimensional assemblies of iron oxide nanocubes with a disordered arrangement are fabricated at the diethylene-glycol/air interface in the presence of assembly fields $(0 / 1 / 3 / 5 / 30 / 50 \mathrm{mT})$. Ring-shaped assemblies gradually transform as the assembly field increases from 0 to $50 \mathrm{mT}$, first to a porous network consisting of elongated assemblies and then to an aligned array of filaments, in which the aligned filaments are formed when the assembly field is $\geq 3 \mathrm{mT}$ and duration $t>14 \mathrm{~min}$. Spin-glass characteristics and static (dynamic) anisotropy factors $\sim 2(3)$ are achieved by tuning the strength of the assembly field. In the presence of a relatively weak assembly field, the interplay between dipolar interactions and disorder with respect to magnetic easy axis alignment leads to spin-glass characteristics. The alignment of the magnetic easy axes and the strength of the dipolar interactions increase with increasing assembly field, resulting in the disappearance of spin-glass characteristics and enhancement of the magnetic anisotropy. This study presents a strategy for obtaining magnetic assemblies with spin-glass behavior and controllable anisotropy while shedding light on the magnetic interactions of low-dimensional assemblies.
\end{abstract}

Keywords: magnetic nanoparticles, assembly, anisotropy, spin glass

\section{INTRODUCTION}

Magnetic nanoparticles and assembled nanostructures have been widely applied in science and technology due to their unique magnetic properties, biocompatibility, and low efficiency. Nowadays, nanoparticles produce assembled nanostructures of various shapes, including spheres, cubes, rods, and tetrapods, with a wide range of array sizes, shapes, and arrangements. The interest in iron oxide nanoparticles, one of the most studied magnetic nanoparticles, is mainly focused on their application in the field of biomedicine $[1,2]$, e.g., magnetic biosensing $[3,4]$, immunoseparation [5], magnetic particle imaging [6], targeted drug delivery [7], and magnetic hyperthermia therapy [8]. Moreover, iron oxide nanostructures are also used in other applications, such as magneto-responsive devices [9], flexible magnetic actuators [10], and electromagnetic devices [11]. Assembly or aggregation of such building blocks yields complex multi-core nanoparticles, so-called nanoflowers or colloidal nanoclusters, and mesocrystal systems with properties that are strongly influenced by interparticle interactions [12-15]. Exposing iron oxide nanoparticle dispersions to a magnetic field leads to the formation of one-dimensional (1D) assemblies with unique magnetic properties [16].

Previous studies show that the orientation and amplitude of the assembly field play a decisive role in the formation of magnetic nanoparticle assemblies. Different 1D arrays of magnetic nanoparticle assemblies, such as rods [17], chains [18], needles [19], and helices [20], can be formed in the presence of magnetic fields parallel to the assembly surface, while hexagonal arrays of pillars $[20,21]$ and labyrinthine patterns [22] can be obtained in a perpendicular magnetic field. Additionally, 1D filaments and helices, as well as C-shaped assemblies, can be achieved by adjusting the direction of the magnetic field with respect to the assembly surface [23]. Contrary to studies focusing on the orientation of the magnetic field, detailed studies of the influence of the magnetic field strength, especially in the low magnetic field range, are few. To the best of our knowledge, assembly fields are in the range of $10-150 \mathrm{mT}[9,19,20,23-29]$ and larger than $200 \mathrm{mT}$ [30-33]. The magnetic interactions, i.e., dipole-dipole interactions, and the alignment of the magnetic easy axes strongly depend on the strength of the assembly field $[20,34]$. Recently, a colossal anisotropy of the dynamic magnetic susceptibility has been reported in $1 \mathrm{D}$ needles assembled on $\mathrm{Si}$ wafers, which is ascribed to a synergistic coupling between a net anisotropy field from a partial easy axis alignment and the dipolar interaction field [19]. These interactions can result in complex magnetic behaviors of assemblies in the low-field range, requiring further clarification. More importantly, the use of a low magnetic field simplifies device design.

Additionally, spin-glass behaviors related to magnetic interactions have also been investigated in low-dimensional systems. Non-interacting magnetic nanoparticles are superparamagnetic

\footnotetext{
${ }^{1}$ College of Physics and Optoelectronic Engineering, Shenzhen University, Shenzhen 518060, China

${ }^{2}$ School of Materials Science and Engineering, Hanshan Normal University, Chaozhou 521041, China

${ }^{3}$ Solid State Physics, Department of Materials Science and Engineering, Ångström Laboratory, Uppsala University, Box 35, 751 03 Uppsala, Sweden

${ }^{4}$ MOE Key Laboratory for Nonequilibrium Synthesis and Modulation of Condensed Matter and State Key Laboratory for Mechanical Behavior of Materials, Xi'an Jiaotong University, Xi'an 710049, China

*Corresponding authors (emails: yjzeng@szu.edu.cn (Zeng YJ); german.salazar.alvarez@angstrom.uu.se (Salazar-Alvarez G))
} 
at high temperatures, while interparticle interactions for dense nanoparticle systems order the nanoparticle magnetic moments below a critical temperature [35]. Spin-glass characteristics from dipole-dipole interactions between the magnetic nanoparticles, such as long relaxation times, magnetic aging, and memory effects, have been reported for dispersions and 2D systems [36 $-41]$. Furthermore, dipolar interactions can lead to long-range ordering of the nanoparticle magnetic moments $[42,43]$.

In this study, by varying the assembly field $(0 / 1 / 3 / 5 / 30 /$ $50 \mathrm{mT}$ ), we systemically studied the magnetic properties of lowdimensional assemblies of iron oxide nanocubes at a liquid-air interface, including the magnetic anisotropy of the static and dynamic magnetic responses. Results show that aligned filaments are formed when the assembly field is $\geq 3 \mathrm{mT}$ and duration $t>14 \mathrm{~min}$. The interplay between dipolar interactions and the alignment of the nanocube magnetic easy axes are strongly affected by the amplitude of the assembly field. Particularly, spin-glass characteristics are observed in the low-field range. This work reports the assembly field required for lowdimensional assemblies and demonstrates that the spin-glass behavior can be switched on and off, varying the strength of the assembly field. Our work contributes to a comprehensive understanding of magnetic interactions of assemblies and provides a strategy for designing multifunctional assemblies in a weak magnetic field range.

\section{EXPERIMENTAL SECTION}

The combination study of liquid and particles refers to the field of nanosynthesis and application, e.g., the liquid phase method [44-46]. Here, oleate-capped iron oxide nanocubes with an edge-length of $l=13.6 \mathrm{~nm}(\mathrm{C} 136)$ were synthesized using a thermal decomposition method and their core-shell structure of $\mathrm{Fe}_{1-x} \mathrm{O} \mid \mathrm{Fe}_{3-\delta} \mathrm{O}_{4}$ with $5 \mathrm{wt} \% \mathrm{Fe}_{1-x} \mathrm{O}$ had been reported [47]. A nanocube dispersion with a concentration of $7 \mathrm{mg} \mathrm{mL}^{-1}$ was prepared by adding cyclohexane and oleic acid. Oriented assemblies were prepared by drop-casting monodisperse oleic acid-capped nanocubes at the air interface of diethylene-glycol (DEG, Aldrich, 99\%) in the presence of a magnetic field. A schematic process of the DEG-air assembly under a magnetic field is shown in Fig. 1a1-a3). Each experiment had a Teflon well (diameter $\sim 2 \mathrm{~cm}$; height $\sim 1 \mathrm{~cm}$ ) placed between the poles of a magnet. A Helmholtz coil with a diameter of $225 \mathrm{~mm}$ was used to generate magnetic fields in the range of $0-5 \mathrm{mT}$, whereas larger magnetic fields were applied with a BRUKER BE-10 electromagnet. DEG of $400 \mu \mathrm{L}$ was added to the well and a Si substrate $\left(4 \times 4 \mathrm{~mm}^{2}\right)$ was positioned at the bottom of the well, followed by adding $8 \mu \mathrm{L}$ of nanocube dispersion at the DEG-air interface. The Teflon well was immediately covered with a glass slide and a constant magnetic field was applied in the plane of the DEG-air interface (Fig. 1a1). A series of assemblies at the DEG-air interface was prepared by applying magnetic fields of different strengths and duration and directly monitored using a light microscope equipped with a digital camera. The freestanding assemblies obtained are transferable to various substrates. Here, the DEG was slowly removed from the bottom of the well with a syringe until the assemblies touched down on the Si substrate (Fig. 1a2). The remaining DEG evaporated in a desiccator under reduced pressure to obtain the assemblies on the Si substrate and the direction of the oriented arrays was along the assembly field direction $H_{\text {ass }}$ as illustrated in Fig. 1a3. An Olympus BX60 upright compound microscope with an
OMAX-A3580U3 digital camera was used to record optical microscope images and videos. The orientational distribution of the assemblies was evaluated using the local gradient orientation method in the directionality routine of Fiji-Image [48]. A normal distribution function was used to fit the histograms and the mean orientation angle, full width at half maximum (FWHM), and average filament width were estimated. Furthermore, the assemblies were imaged using a Zeiss-LEO 1550 scanning electron microscope (SEM) at an accelerating voltage of $20 \mathrm{kV}$. Atomic force microscopy (AFM) images were collected using a Bruker MultiMode 8-HR atomic force microscope to determine the dimensions of the iron oxide nanocube assemblies. To observe the arrangement of iron oxide nanocubes inside assemblies, sliced cross-section samples were prepared using an FEI Scios DualBeam FIB-SEM. The morphologies of iron oxide nanocube assemblies were further investigated using a JEOL transmission electron microscope (TEM) F200 operating at a $200 \mathrm{kV}$ accelerating voltage. The angle-dependent magnetization measurements were performed using a LakeShore 7404 VSM equipped with a sample rotation stage. Quasi-static (DC) and dynamic (AC) magnetization measurements were performed in a quantum design MPMS-XL superconducting quantum interference device magnetometer equipped with the ultra-low-field option.

\section{RESULTS AND DISCUSSION}

Iron oxide nanocubes assemble at the DEG-air interface in the presence of an external magnetic field. The fabrication process is schematically shown in Fig. 1a. Optical microscope images of the assembled nanocubes under various assembly fields $(0-50 \mathrm{mT})$ were recorded at the DEG-air interface (Fig. 1b). The assemblies evolve from an isotropic ring-shaped porous network in the absence of a field toward an oriented array. Remarkably, the alignment is visible at a low field of $3 \mathrm{mT}$, where the particles begin to assemble in long intertwined filaments. Fig. 1c, d show the data of the orientational analysis of the images and the image of the 50-mT assemblies, respectively. The data are summarized as orientation histograms and fitted using a normal distribution. An increase in the assembly field increases the alignment of the filaments along the assembly field direction $H_{\text {ass }}$. Further, the distribution of orientations becomes narrower (smaller FWHM, see Fig. 1c). The onset field $3 \mathrm{mT}$ for the alignment is lower than that in earlier reports involving nanocubes with similar sizes on silicon substrates $(65 \mathrm{mT})$ [19] and at the DEG-air interface $(17 \mathrm{mT})$ [20]. At a high assembly field, the DEG-air interface adjusts the assembly morphology to reduce the magnetostatic self-energy of the assembly, yielding low-dimensional curved structures as opposed to $1 \mathrm{D}$ needle-shaped structures in the assembly on Si substrates. These results suggest that the interactions of the iron oxide nanocubes with the Si substrate are not negligible due to the less freedom to rotate [49]; the duration of the applied field $(t)$ during solvent evaporation can be relevant. Previous investigations indicate that the structural evolution, ordering, and positioning of assemblies are controlled by $t$ [24,27]. Applying a field of $5 \mathrm{mT}$ results in an isotropic network at $t=0$ to $12 \mathrm{~min}$; however, for longer durations, the alignment is as shown in Fig. S1a. Even larger magnetic fields cannot induce the alignment at $t=12 \mathrm{~min}$ (Fig. S1b). Two real-time videos for $0 / 1 \mathrm{mT}$ assemblies were recorded to investigate the evolution of the assembly process and video clips with a time range of 13-15 min are correspondingly supplemented as Videos 


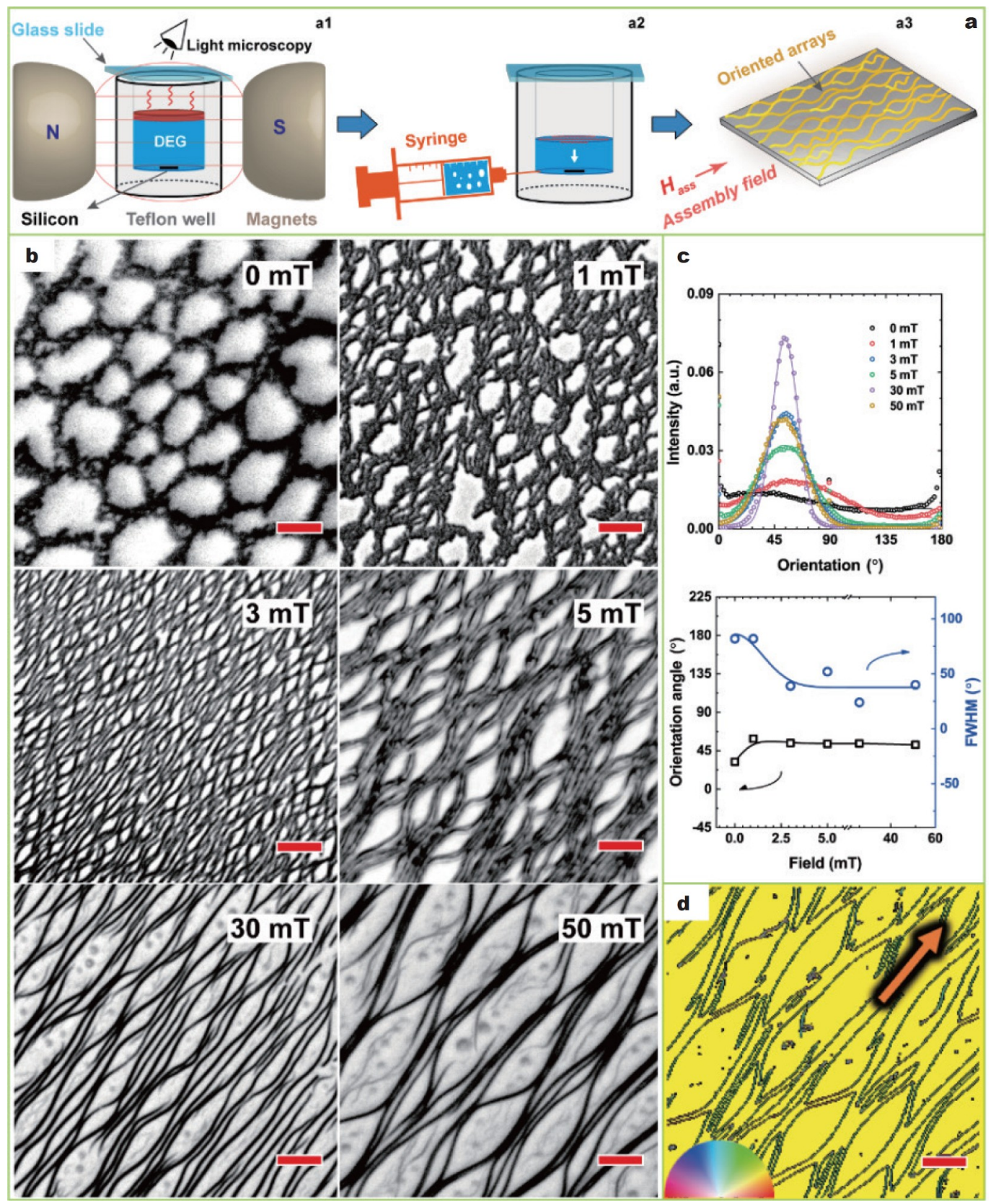

Figure 1 Oriented assembly of nanocubes under an assembly magnetic field. (a) Schematic illustration of the fabrication of assemblies at the DEG-air interface and transfer onto Si substrates. (b) Optical microscope images showing the length and shape of 0/1/3/5/30/50 mT assemblies at the DEG-air interface. (c) Distribution of the orientations of the filaments (top) and data of the Gaussian fits (bottom). (d) Example of the distribution of orientations of the sample prepared with an assembly field of $50 \mathrm{mT}$. The arrow represents the direction of the assembly field and the scale bars are $20 \mu \mathrm{m}$.

SV1 and SV2. Results show that the nanocubes assemble into straight arrays at the DEG-air interface when $t$ is $\sim 14$ min. As $t$ increases, these assemblies gradually turn into curved filaments. After $t \sim 18 \mathrm{~min}$, no changes are observed on assemblies. These results demonstrate that the evaporation rate of cyclohexane and the magnetic field conjointly are responsible for the formation of aligned arrays. Hereafter, assemblies fabricated at $t=25 \mathrm{~min}$ with different strengths of the assembly field are discussed.

The average filament width was obtained using the Gaussian curve fitting from the histograms. Fig. 2a shows that the average filament width reduced rapidly when the applied field changed from 0 to $3 \mathrm{mT}$, while it decreased slowly as the applied field further increased. To characterize the mesoscale structure of assemblies, SEM, AFM, and TEM of 5-mT assemblies are shown in Fig. 2b-e. Upon completing the transfer from DEG to the Si substrate, the structure of the oriented assemblies remains the same (Fig. S2 and Fig. 2c). The SEM image in Fig. 2b indicates a disordered arrangement of iron oxide nanocubes on the top surface which was further verified using the variation of the AFM height profile recorded along the length of the nanorod (Fig. 2d). Fig. 2e shows a TEM image of the cross-section with maze-like patterns with one nanocube having an edge length of $13.6 \mathrm{~nm}$ presented in the left inset. The image indicates that filaments are composed of locally disordered nanocubes, as 

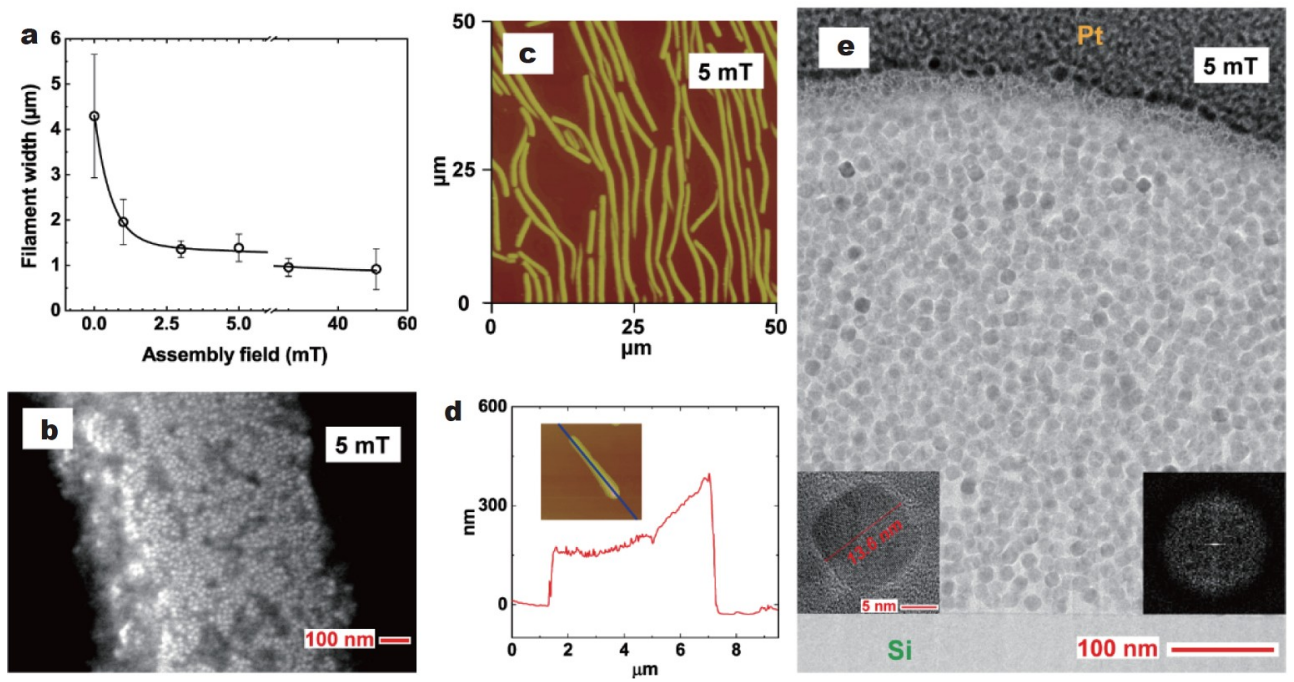

(c) AFM topography image, (d)
ight insets of (e) show the TEM AFM height trace along the blue line in the inset, and (e) TEM image of the cross-section of 5-mT assemblies. The left and right insets of (e) show the TEM image of an iron oxide nanocube and the fast Fourier transform pattern of the cross-section, respectively. Scale bars (red): 100 nm.

evidenced by the halo in the fast Fourier transform image (see right inset). Similarly, disordered mesoscale structures of 0/50$\mathrm{mT}$ assemblies are shown in Figs S3 and S4. Remarkably, with increasing assembly field, the thickness of assemblies increases (Fig. S4), consistent with the reduction in the average filament width. These observations indicate that the amorphous aggregation of nanocrystals is determined by the strength of the assembly field, where the morphologies and magnetic interactions of assemblies are tuned. Contrary to the ordered structures, i.e., $2 \mathrm{D}$ mesocrystals with a simple cubic lattice $(0 \mathrm{mT})$ [29] and $1 \mathrm{D}$ needles with a body-centered tetragonal structure $(65 \mathrm{mT})$ [19], this amorphous structure results from the absence of excess oleic acid in dispersions [20].

Fig. 3 shows the DC-magnetic properties of the assemblies transferred to silicon substrates. The low-field magnetization curves of samples and the measuring field parallel to the assembly field $H_{\text {ass }}$, are shown in Fig. 3a, whereas Fig. 3b shows the normalized magnetization values $M / M_{\mathrm{S}}$ of the samples as a function of the angle between the measuring and assembly fields. The details of normalized method are illustrated in Fig. S5. The 50-mT sample measured both parallel and perpendicular to the assembly field exhibits significant anisotropy of the magnetization at low fields. This is consistent with previous results of needle-shaped arrays [19]. For the measuring field parallel to the direction of the assembly field $H_{\text {ass }}$, with increasing strength of the assembly field, the magnetization hysteresis $(M-H)$ curves display an increasing magnetization and a more distinct nonlinear magnetic response, proving a significant enhancement of the anisotropy.

Fig. $3 \mathrm{~b}$ shows the angle-dependent magnetization curves of $0 /$ $1 / 3 / 5 / 30 / 50-\mathrm{mT}$ assemblies at room temperature for a measuring field of $5 \mathrm{mT}$. No angular dependence is exhibited by $0-\mathrm{mT}$ assemblies (straight line), representing an isotropic magnetic response. However, other assemblies exhibit a sinusoidal angular dependence. To quantify the anisotropy of the DC-magnetic response, we introduce the static anisotropy factor $A_{\mathrm{DC}}$ using the ratio of parallel to perpendicular magnetization, i.e., $A_{\mathrm{DC}}=M_{\|} /$ $M_{\perp}$, where $M_{\|}$and $M_{\perp}$ are the maximum and minimum values of the angular-dependent magnetization, respectively, indicated by arrows in Fig. 3b. The assembly field-dependent behaviors of $A_{\mathrm{DC}}$ for the $0 / 1 / 3 / 5 / 30 / 50-\mathrm{mT}$ assemblies are shown in the inset of Fig. 3b. $A_{\mathrm{DC}}$ rapidly increases from 1 to $\sim 1.5$ when the assembly field is increased to $5 \mathrm{mT}$, while a gradual but weak increase occurs with a further increase of the assembly field. A static anisotropy factor $A_{\mathrm{DC}} \sim 2.0$ is obtained for an assembly field of $50 \mathrm{mT}$. Essentially, applying small assembly fields of 1/3/ $5 \mathrm{mT}$ is sufficient to achieve a considerable degree of magnetic anisotropy, revealing that the nanocubes are highly sensitive to the assembly field applied over the DEG-air interface.

AC magnetization measurements were performed on the $0 / 3$ / $5 / 50-\mathrm{mT}$ assemblies in a temperature range of $50-300 \mathrm{~K}$ using AC field frequencies of $0.17-510 \mathrm{~Hz}$, as shown in Fig. S6. In all cases, the amplitude of the AC magnetic field was $0.4 \mathrm{mT}$ and it was parallel toward the assembly field $H_{\text {ass. }}$ The in-phase $\left(M^{\prime}\right)$ and out-of-phase $\left(M^{\prime \prime}\right)$ components of the AC magnetization recorded with an assembly field of $50 \mathrm{mT}$ are shown in Fig. 4a. The $M^{\prime}$ and $M^{\prime \prime}$ versus temperature curves of the 0/5/50-mT assemblies at AC field frequency $f=0.17 \mathrm{~Hz}$ are compared in Fig. 4b. Results show that the $M^{\prime}$ and $M^{\prime \prime}$ versus temperature curves increase significantly in magnitude as the assembly field increases from 0 to $50 \mathrm{mT}$. The AC magnetization for $50-\mathrm{mT}$ assemblies with the measuring field perpendicular to the $H_{\text {ass }}$ was also measured, as shown in Fig. S7. To quantify the anisotropy of the AC magnetization, the temperature-dependent anisotropy factor $A_{\mathrm{AC}}$ was defined as the ratio of parallel to perpendicular in-phase magnetization components. The calculated $A_{\mathrm{AC}}$ versus temperature curve for the $50-\mathrm{mT}$ assembly is shown in Fig. $4 \mathrm{c}$, with the peak value of $A_{\mathrm{AC}} \sim 3$. We compared this result with that obtained for 1D C136 needles in [19], where a maximum anisotropy factor as large as 80 was obtained. With the same $M^{\prime}\left(\| H_{\text {ass }}\right)$ magnitude for needles and filaments, the large difference in anisotropy factor is caused by the larger $M^{\prime}$ $\left(\perp H_{\text {ass }}\right)$ of 50 -mT assemblies compared with that of needles.

The influence of the assembly field on the relaxation properties of assemblies was investigated by analyzing the peak temperature $T_{\mathrm{p}}$ of the in-phase component of the AC magnetization 

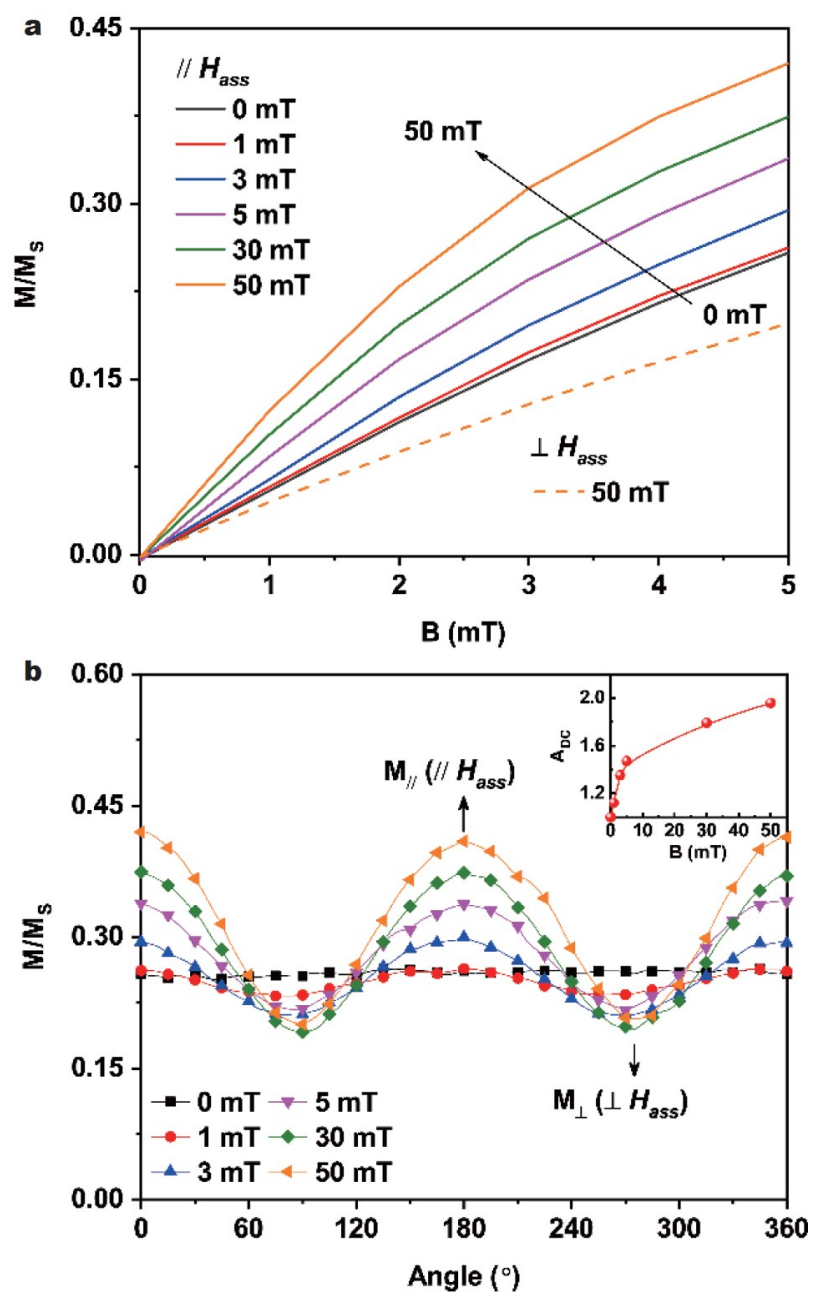

Figure 3 Low-field DC-magnetic properties of iron oxide nanocubes assembled in various magnetic fields. (a) Low-field magnetization curves of $0 /$ $1 / 3 / 5 / 30 / 50-\mathrm{mT}$ assemblies with the measuring field parallel to the assembly field direction $H_{\text {ass. }}$. The dotted line represents the magnetization obtained with the measuring field perpendicular to $H_{\text {ass. }}$ (b) Angle-dependent magnetization of 0/1/3/5/30/50-mT assemblies with a measuring magnetic field of $5 \mathrm{mT}$. Inset shows the assembly field dependence of the static anisotropy factor $A_{\mathrm{DC}}$.

as a function of AC field frequency $f$. Previous studies show that the critical slowing down law, $\tau=2 \pi f=\tau_{0}\left(T_{\mathrm{p}} / T_{\mathrm{g}}-1\right)^{-z v}$, can be used to show critical slowing down of the relaxation time $(\tau)$ as the spin-glass transition temperature $\left(T_{\mathrm{g}}\right)$ is approached, where $\tau_{0}$ and $z v$ are a microscopic timescale related to the relaxation of the individual particle moments and the dynamic critical exponent, respectively. Fig. $4 \mathrm{~d}$ shows $\ln (\tau)$ versus $T_{\mathrm{p}}$ for the $0 / 3$ / $5 / 50-\mathrm{mT}$ assemblies together with fits to the critical slowing down law. Frozen ferrofluids can exhibit spin-glass behaviors when the microscopic relaxation time $\tau_{0}$ ranges from $10^{-9}-10^{-8} \mathrm{~s}$ $[36,37]$. Only the results for the $3 / 5-\mathrm{mT}$ assemblies agree with expectations for a spin-glass system, yielding the fitting parameters $\tau_{0}=1.6 \times 10^{-9} \mathrm{~s}, z v=12, T_{\mathrm{g}}=175 \mathrm{~K}$ and $\tau_{0}=1.0 \times 10^{-8} \mathrm{~s}$, $z v=6.8, T_{\mathrm{g}}=202 \mathrm{~K}$, respectively, while for the other assemblies, low values of $\tau_{0}$ are obtained. Thus, these results prove that the spin-glass behavior occurs in assemblies formed using a narrow range of small assembly fields.

To better understand the evolution of the magnetic anisotropy and the appearance of spin-glass behavior with increasing assembly fields, schematic drawings of C136 nanocube assemblies with varying assembly fields are illustrated in Fig. 5. The C136 nanocubes are superparamagnetic at room temperature, in which the magnetic easy axes correspond to different [111] crystallographic directions (schematically plotted by connecting two diagonally opposite corners in Fig. 5a), and the nanocube magnetic moments in the superparamagnetic state fluctuate in different [111] directions. For simplicity, we ignore possible contributions to the magnetic anisotropy from shape anisotropy. From previous investigations [9,30], the magnetic dipole moment of a particle is defined by $m=\mu_{0} \chi V\left(H+H_{\text {dip }}\right)$, where $\mu_{0}$, $\chi, V, H$ and $H_{\text {dip }}$ denote magnetic permeability of free space, superparamagnetic susceptibility, nanoparticle volume, assembly magnetic field, and dipolar interaction field, respectively. The magnetic dipole-dipole interaction energy between two particles with aligned dipole moments can be expressed as $\mu=-\left(\mathrm{m}^{2} /\right.$ $\left.4 \pi \mu_{0} r^{3}\right)\left(3 \cos ^{2} \theta-1\right)$, where $r$ is the distance between the particles and $\theta$ is the angle between $\vec{r}$ and $\vec{m}$. From Fig. 5a, two nanocubes will have negative interaction energy (attractive force) when $\theta=0^{\circ}$ and positive interaction energy (repulsive force) when $\theta=90^{\circ}$. Therefore, the degree of aligned assemblies and average filament width, to some extent, provides a blueprint of the distribution of dipolar interactions, depending solely on the strength of the assembly field.

The zero-assembly field case reveals a porous network of ringshaped assemblies formed by dipolar fields due to a few larger nanocubes with blocked magnetic moments at room temperature. These interaction fields, in turn, magnetize smaller (superparamagnetic) nanocubes. Also, the lowest energy state for the zero-assembly field becomes the assembly geometries forming closed magnetic circuits to minimize the magnetostatic self-energy (Fig. 5b, left). In the presence of a relatively weak assembly field $(1 / 3 / 5 \mathrm{mT})$, ring-shaped assemblies due to the Zeeman energy and the magnetic torque exerted by the assembly field break up to form more low-dimensional filament structures along the assembly field direction. It also simultaneously introduces the distribution of dipole-dipole interactions, favoring parallel and anti-parallel alignment of the nanocube magnetic moments. Previous simulations show that the dipoles assumed a zigzag configuration unlocked from an assembly field of $16.7 \mathrm{mT}$ [20]. The distribution of magnetic interactions is largely determined by the disorder in the alignment of the nanocube magnetic easy axes, yielding magnetic disorder and frustration (Fig. 5b, middle). For the larger assembly fields $(30 / 50 \mathrm{mT})$, the aligned filaments indicate a reduction in the width of the distribution of dipole-dipole interactions. The larger fields also rotate the magnetization easy axes of the nanocubes toward the assembly field. A synergistic interplay between dipole interactions and magnetic easy axes establishes the 1D geometry shown in Fig. 5b (right). Electron holography reveals the existence of dipolar ferromagnets in magnetic field-induced 1D structures without crystallinity [33]. These results show that an increase in the assembly field facilitates the anisotropic growth of assemblies by dipolar interactions and the alignment of the nanocube magnetic easy axes along the assembly field direction. Particularly, changing the distribution of interactions between $0-5 \mathrm{mT}$ and 5-50 $\mathrm{mT}$ leads to the change of the static anisotropy factor $A_{\text {DC }}$ shown in the inset of Fig. $3 \mathrm{~b}$.

Magnetic systems should have two characteristics, namely magnetic disorder and frustration, to display spin-glass beha- 

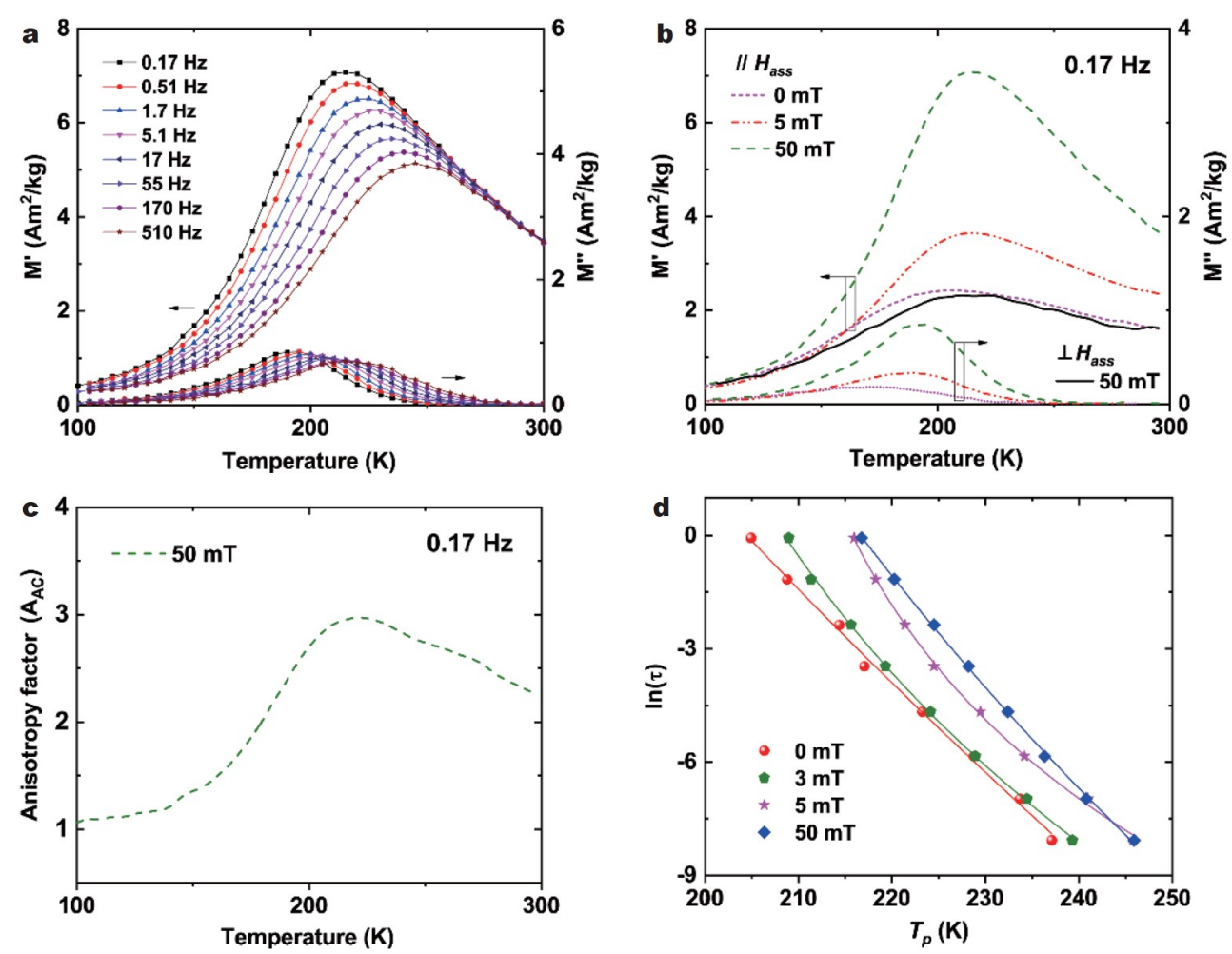

Figure 4 (a) In-phase $\left(M^{\prime}\right)$ and out-of-phase $\left(M^{\prime}\right)$ components of the AC magnetization versus temperature for different AC frequencies and an assembly field of $50 \mathrm{mT}$. (b) Comparison of $M^{\prime}$ and $M^{\prime \prime}$ at $f=0.17 \mathrm{~Hz}$ for $0 / 5 / 50-\mathrm{mT}$ assemblies with the measuring field parallel to the direction of the assembly field $H_{\text {ass }}$. The solid line represents $M^{\prime}$ obtained using the measuring field perpendicular to $H_{\text {ass. }}$. (c) Anisotropy factor $\left(A_{\mathrm{AC}}\right)$ versus temperature $(f=0.17 \mathrm{~Hz})$ for the 50-mT assemblies. (d) $\ln \tau$ versus peak-temperature $T_{\mathrm{p}}$ for the $0 / 3 / 5 / 50-\mathrm{mT}$ assemblies. Solid lines correspond to the critical slowing down law (see text for details).
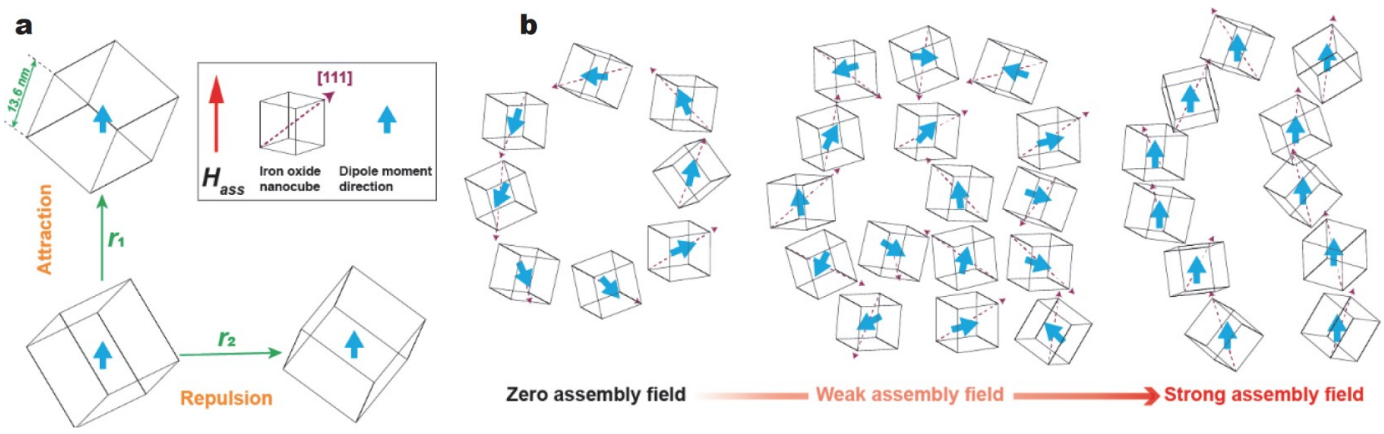

Figure 5 Schematic representation of assemblies formed under different strengths of the assembly field. (a) Magnetic dipole-dipole interactions between nanoparticles induced by an external magnetic field. The blue and red arrows indicate the direction of the nanocube magnetic moment and the direction of the assembly field $H_{\text {ass, }}$, respectively. (b) Assembly geometries and direction of nanocube magnetic moments inside assemblies under zero, weak, and strong assembly fields.

viors. A previous study indicates that characteristics of a spinglass are largely missing in the $\mathrm{C} 136$ nanocube platelets, while it exists in the C136 nanocube paste [19]. Further comparison shows that the C136 nanocube platelets have an ordered structure of nanocubes and the $\mathrm{C} 136$ nanocube paste is composed of disordered assemblies of C136 nanocubes. Disordered assemblies with a disordered alignment of nanocube easy axes lead to magnetic disorder and frustration, responsible for the spin-glass behavior of $\mathrm{C} 136$ nanocube paste. In this study, the interplay between dipole-dipole interactions and disordered alignment of the magnetic easy axes in the presence of a small assembly field $(3 / 5 \mathrm{mT})$ results in magnetic disorder and frustration (Fig. $5 \mathrm{~b}$, middle). However, the alignment of the magnetic easy axes under a larger assembly field $(30 / 50 \mathrm{mT})$ forms ordered magnetic interactions (Fig. 5b, right). Theoretically, the lower critical dimension for a low-temperature spin-glass phase is somewhere in the range of 2-3 [50], implying that no phase transition is allowed at a finite temperature below this dimension. Thus, spin-glass characteristics can occur in 3D porous networks of 3/ $5-\mathrm{mT}$ assemblies and disappear for the 1D filaments of 30/50$\mathrm{mT}$ assemblies. Our work reveals that the assemblies associated with magnetic interactions are tuned using the strength of the assembly field.

\section{CONCLUSIONS}

We have systemically studied the properties of the magnetic 
field-induced iron oxide nanocube assemblies at the DEG-air interface. Results show that the evaporation rate of cyclohexane and the amplitude of the assembly field determine the geometry of the assemblies. Furthermore, two critical parameters are responsible for the aligned filaments, namely, the onset field $\geq 3 \mathrm{mT}$ and the duration $t>14 \mathrm{~min}$. The interplay between dipolar interactions and the magnetic easy axes of nanocubes is tuned using the strength of the assembly field. The ring-shaped assemblies observed for zero-assembly field are gradually transformed by increasing the assembly field from 0 to $50 \mathrm{mT}$, first to a disordered network intertwined filament-like assemblies and then to $1 \mathrm{D}$ assembly filaments with enhanced static and dynamic anisotropic factors. Moreover, the interplay between dipolar interactions and magnetic easy axes in the presence of a relatively weak assembly field causes magnetic disorder and frustration, resulting in spin-glass characteristics. Additionally, the spin-glass characteristics can be induced by weak assembly fields, which are masked in the presence of a large assembly field.

\section{Received 30 March 2021; accepted 28 May 2021; published online 21 July 2021}

1 Pankhurst QA, Connolly J, Jones SK, et al. Applications of magnetic nanoparticles in biomedicine. J Phys D-Appl Phys, 2003, 36: R167R181

2 Pankhurst QA, Thanh NTK, Jones SK, et al. Progress in applications of magnetic nanoparticles in biomedicine. J Phys D-Appl Phys, 2009, 42: 224001

3 Tian B, Liao X, Svedlindh P, et al. Ferromagnetic resonance biosensor for homogeneous and volumetric detection of DNA. ACS Sens, 2018, 3: 1093-1101

4 Issadore D, Park YI, Shao H, et al. Magnetic sensing technology for molecular analyses. Lab Chip, 2014, 14: 2385-2397

5 Miltenyi S, Müller W, Weichel W, et al. High gradient magnetic cell separation with macs. Cytometry, 1990, 11: 231-238

6 Dieckhoff J, Kaul MG, Mummert T, et al. Magnetic particle imaging of liver tumors in small animal models. Int J Mag Part Imag, 2017, 3: 1707003

7 Felfoul O, Mohammadi M, Taherkhani S, et al. Magneto-aerotactic bacteria deliver drug-containing nanoliposomes to tumour hypoxic regions. Nat Nanotech, 2016, 11: 941-947

8 Attaluri A, Kandala SK, Wabler M, et al. Magnetic nanoparticle hyperthermia enhances radiation therapy: A study in mouse models of human prostate cancer. Int J Hyperthermia, 2015, 31: 359-374

9 He L, Wang M, Ge J, et al. Magnetic assembly route to colloidal responsive photonic nanostructures. Acc Chem Res, 2012, 45: 1431-1440

10 Olsson RT, Azizi Samir MAS, Salazar-Alvarez G, et al. Making flexible magnetic aerogels and stiff magnetic nanopaper using cellulose nanofibrils as templates. Nat Nanotech, 2010, 5: 584-588

11 Yun H, Liu X, Paik T, et al. Size- and composition-dependent radio frequency magnetic permeability of iron oxide nanocrystals. ACS Nano, 2014, 8: 12323-12337

12 Schaller V, Wahnström G, Sanz-Velasco A, et al. Monte Carlo simulation of magnetic multi-core nanoparticles. J Magn Magn Mater, 2009, 321: $1400-1403$

13 Lartigue L, Hugounenq $\mathrm{P}$, Alloyeau D, et al. Cooperative organization in iron oxide multi-core nanoparticles potentiates their efficiency as heating mediators and MRI contrast agents. ACS Nano, 2012, 6: 1093510949

14 Bender P, Fock J, Frandsen C, et al. Relating magnetic properties and high hyperthermia performance of iron oxide nanoflowers. J Phys Chem C, 2018, 122: 3068-3077

15 Faure B, Wetterskog E, Gunnarsson K, et al. 2D to 3D crossover of the magnetic properties in ordered arrays of iron oxide nanocrystals. Nanoscale, 2013, 5: 953-960
16 Lisjak D, Mertelj A. Anisotropic magnetic nanoparticles: A review of their properties, syntheses and potential applications. Prog Mater Sci, 2018, 95: 286-328

17 Pileni MP. Nanocrystal self-assemblies: Fabrication and collective properties. J Phys Chem B, 2001, 105: 3358-3371

18 Toulemon D, Rastei MV, Schmool D, et al. Enhanced collective magnetic properties induced by the controlled assembly of iron oxide nanoparticles in chains. Adv Funct Mater, 2016, 26: 2454-2462

19 Wetterskog E, Jonasson C, Smilgies DM, et al. Colossal anisotropy of the dynamic magnetic susceptibility in low-dimensional nanocube assemblies. ACS Nano, 2018, 12: 1403-1412

20 Singh G, Chan H, Baskin A, et al. Self-assembly of magnetite nanocubes into helical superstructures. Science, 2014, 345: 1149-1153

21 Dikansky YI, Zakinyan AR, Mkrtchyan LS. Instability of a thin layer of a magnetic fluid in a perpendicular magnetic field. Tech Phys, 2010, 55: $1270-1274$

22 Zhang Q, Janner M, He L, et al. Photonic labyrinths: Two-dimensional dynamic magnetic assembly and in situ solidification. Nano Lett, 2013, 13: $1770-1775$

23 Singh G, Chan H, Udayabhaskararao T, et al. Magnetic field-induced self-assembly of iron oxide nanocubes. Faraday Discuss, 2015, 181: 403-421

24 Fang WX, He ZH, Xu XQ, et al. Magnetic-field-induced chain-like assembly structures of $\mathrm{Fe}_{3} \mathrm{O}_{4}$ nanoparticles. Europhys Lett, 2007, 77: 68004

25 Jestin J, Cousin F, Dubois I, et al. Anisotropic reinforcement of nanocomposites tuned by magnetic orientation of the filler network. Adv Mater, 2008, 20: 2533-2540

26 Li L, Yang Y, Ding J, et al. Synthesis of magnetite nanooctahedra and their magnetic field-induced two-/three-dimensional superstructure. Chem Mater, 2010, 22: 3183-3191

27 Fragouli D, Buonsanti R, Bertoni G, et al. Dynamical formation of spatially localized arrays of aligned nanowires in plastic films with magnetic anisotropy. ACS Nano, 2010, 4: 1873-1878

28 Yao Y, Metwalli E, Niedermeier MA, et al. Nano- and microstructures of magnetic field-guided maghemite nanoparticles in diblock copolymer films. ACS Appl Mater Interfaces, 2014, 6: 5244-5254

29 Wetterskog E, Klapper A, Disch S, et al. Tuning the structure and habit of iron oxide mesocrystals. Nanoscale, 2016, 8: 15571-15580

30 Park JI, Jun Y, Choi J, et al. Highly crystalline anisotropic superstructures via magnetic field induced nanoparticle assembly. Chem Commun, 2007, 5001

31 Ahniyaz A, Sakamoto Y, Bergström L. Magnetic field-induced assembly of oriented superlattices from maghemite nanocubes. Proc Natl Acad Sci USA, 2007, 104: 17570-17574

32 Sahoo Y, Cheon M, Wang S, et al. Field-directed self-assembly of magnetic nanoparticles. J Phys Chem B, 2004, 108: 3380-3383

33 Varón M, Beleggia M, Kasama T, et al. Dipolar magnetism in ordered and disordered low-dimensional nanoparticle assemblies. Sci Rep, 2013, 3: 1234

34 Ngo AT, Pileni MP. Assemblies of ferrite nanocrystals: Partial orientation of the easy magnetic axes. J Phys Chem B, 2001, 105: 53-58

35 Bedanta S, Kleemann W. Supermagnetism. J Phys D-Appl Phys, 2008, 42: 013001

36 Djurberg C, Svedlindh P, Nordblad P, et al. Dynamics of an interacting particle system: Evidence of critical slowing down. Phys Rev Lett, 1997, 79: 5154-5157

37 Andersson JO, Djurberg C, Jonsson T, et al. Monte Carlo studies of the dynamics of an interacting monodispersive magnetic-particle system. Phys Rev B, 1997, 56: 13983-13988

38 Sun Y, Salamon MB, Garnier K, et al. Memory effects in an interacting magnetic nanoparticle system. Phys Rev Lett, 2003, 91: 167206

39 Sasaki M, Jönsson PE, Takayama $\mathrm{H}$, et al. Aging and memory effects in superparamagnets and superspin glasses. Phys Rev B, 2005, 71: 104405

40 Poddar P, Telem-Shafir T, Fried T, et al. Dipolar interactions in twoand three-dimensional magnetic nanoparticle arrays. Phys Rev B, 2002, 66: 060403

41 Farrell D, Cheng Y, McCallum RW, et al. Magnetic interactions of iron 
nanoparticles in arrays and dilute dispersions. J Phys Chem B, 2005, 109: 13409-13419

42 Majetich SA, Sachan M. Magnetostatic interactions in magnetic nanoparticle assemblies: Energy, time and length scales. J Phys D-Appl Phys, 2006, 39: R407-R422

43 Morup S, Tronc E. Superparamagnetic relaxation of weakly interacting particles. Phys Rev Lett, 1994, 72: 3278-3281

44 Fang Z, Zhen YR, Neumann O, et al. Evolution of light-induced vapor generation at a liquid-immersed metallic nanoparticle. Nano Lett, 2013, 13: $1736-1742$

45 Wang Z, Li Z, Sun Z, et al. Visualization nanozyme based on tumor microenvironment "unlocking" for intensive combination therapy of breast cancer. Sci Adv, 2020, 6: eabc8733

46 Tang H, Wei J, Liu F, et al. Strong metal-support interactions between gold nanoparticles and nonoxides. J Am Chem Soc, 2016, 138: 56-59

47 Wetterskog E, Agthe M, Mayence A, et al. Precise control over shape and size of iron oxide nanocrystals suitable for assembly into ordered particle arrays. Sci Tech Adv Mater, 2014, 15: 055010

48 Schneider CA, Rasband WS, Eliceiri KW. NIH Image to ImageJ: 25 years of image analysis. Nat Methods, 2012, 9: 671-675

49 Faure B, Salazar-Alvarez G, Bergstrom L. Hamaker constants of iron oxide nanoparticles. Langmuir, 2011, 27: 8659-8664

50 Maiorano A, Parisi G. Support for the value $5 / 2$ for the spin glass lower critical dimension at zero magnetic field. Proc Natl Acad Sci USA, 2018, 115: 5129-5134

Acknowledgements The present work was financially supported by Shenzhen Science and Technology Project (CYJ20180507182246321 and JCYJ20200109105825504) and Swedish Research Council VR (2016-06959). Xiaoqi Liao is grateful for financial support from the Doctoral Joint-Training Program of China Scholarship Council.

Author contributions Liao X, Wetterskog E and Zeng YJ designed the experiments; Liao X, Gunnarsson K and Svedlindh P performed the experiments; Ulusoy S, Liang $\mathrm{H}$ and Huang R performed the data analysis; Liao X, Salazar-Alvarez G, Wang Y, Zeng YJ, and Svedlindh P wrote and revised the manuscript. All authors have discussed and approved the results and conclusions.

Conflict of interest The authors declare no conflict of interest.

Supplementary information Supporting data are available in the online version of the paper.

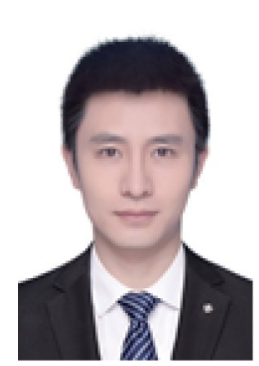

Xiaoqi Liao is a postdoctoral researcher at Shenzhen University. He received his $\mathrm{PhD}$ degree in 2019 from Xi'an Jiaotong University. During 2017-2018, he was a joint-training $\mathrm{PhD}$ student at Uppsala University. His research interest focuses on magnetic materials and devices, including magnetic shape memory alloys, self-assembly of magnetic nanoparticles, and 2D materials.

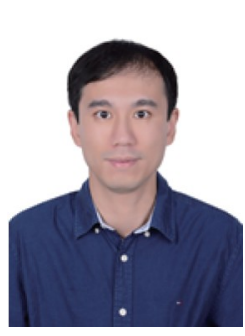

Yu-Jia Zeng is a professor at Shenzhen University. He received his bachelor's degree in materials science and engineering and his $\mathrm{PhD}$ degree in materials physics and chemistry from Zhejiang University. After graduation, he worked at the Department of Physics of $\mathrm{KU}$ Leuven as a postdoctoral fellow and a research associate. His research interests include low-dimensional materials, in particular semiconductors, for optoelectronics, spintronics, and multiferroics.

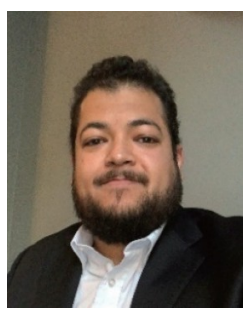

Germán Salazar-Alvarez obtained his $\mathrm{PhD}$ degree in 2005 from the Royal Institute of Technology and his Docent degree from Stockholm University in 2012, where he was a group leader between 2010-2019. Since 2020 he is a senior lecturer at Uppsala University. His research activities are devoted to the synthesis, self-assembly, and characterization of nanomaterials with magnetic, catalytic, and ion transport functionalities.

\section{低场诱导液-气界面纳米颗粒组装体自旋玻璃行为和 可控各向异性}

廖晓奇 ${ }^{1,2,3}$, Seda Ulusoy ${ }^{3}$, 黄锐 ${ }^{2}$, Erik Wetterskog ${ }^{3}$,

Klas Gunnarsson ${ }^{3}$, 王宇 ${ }^{4}$, 梁华伟 ${ }^{1}$, 曾昱嘉 ${ }^{*}$,

German Salazar-Alvarez ${ }^{3 *}$, Peter Svedlindh ${ }^{3}$

摘要 利用纳米尺度结构单元堆积成具有丰富物理性能的一维(1D)组 装体, 是材料设计的研究前沿. 然而, 弱磁场诱导 $1 \mathrm{D}$ 阵列的制备及其磁 性能机制的揭示仍然具有挑战性. 本文采用强度不同的组装场 $(0 / 1 / 3 / 5 /$ $30 / 50 \mathrm{mT}$ ), 在二甘醇-空气界面上制备了由无序四氧化三铁纳米立方 体组成的低维组装体. 随着组装场从0增长到 $50 \mathrm{mT}$, 环形组装体先变 为细长多孔网状, 再逐步变为细丝阵列, 其中细丝阵列的合成条件为组 装场 $\geq 3 \mathrm{mT}$ 且施加组装场时间 $t>14 \mathrm{~min}$. 通过调控组装场强度可得到自 旋玻璃特性和静(动)各向异性 2(3). 在弱组装场下磁偶极和无序排列 易磁化轴之间相互作用导致自旋玻璃特性，进一步增大组装场将加强 磁偶极作用和易磁化轴排列, 导致自旋玻璃特性消失和强各向异性. 本 研究不仅提出了一种制备自旋玻璃和可控各向异性组装体的策略, 还 为理解低维组装磁相互作用提供了新思路. 